\title{
Constant-sized correlations are sufficient to self-test maxi- mally entangled states with unbounded dimension
}

\author{
Honghao Fu
}

Joint Center for Quantum Information and Computer Science, Institute for Advanced Computer Studies and Department of Computer Science, University of Maryland, College Park, MD 20742, USA

Let $p$ be an odd prime and let $r$ be the smallest generator of the multiplicative group $\mathbb{Z}_{p}^{*}$. We show that there exists a correlation of size $\Theta\left(r^{2}\right)$ that selftests a maximally entangled state of local dimension $p-1$. The construction of the correlation uses the embedding procedure proposed by Slofstra (Forum of Mathemat-

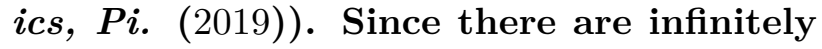
many prime numbers whose smallest multiplicative generator is in the set $\{2,3,5\}$ ( D.R. Heath-Brown The Quarterly Journal of Mathematics (1986) and M. Murty The Mathematical Intelligencer (1988)), our result implies that constant-sized correlations are sufficient for self-testing of maximally entangled states with unbounded local dimension.

The certification of a quantum device is an important building block for many quantum information processing tasks, especially when the devices are provided by some untrusted vendor. We would like such certification to be done based solely on the observed measurement statistics and with the only assumption that any local device cannot communicate with the other local devices. The measurement statistics are referred to as correlations. It has been shown that certain quantum correlations require the distant parties to share a particular entangled state up to some local isometry. This phenomenon is referred to as self-testing.

The fact that the verifier only interacts classically with the unknown device makes self-testing a powerful tool for applications in quantum cryptography and computational complexity theory. It allows a classical party to delegate quantum computations to some untrusted service provider and verify that the computations are performed Honghao Fu: h7fu@umd.edu honestly and correctly [9, 25]. Self-testing also becomes a critical component of the security proofs of device-independent quantum cryptographic protocols [12, 18]. Since the setting of multi-prover interactive proof systems (MIP) is similar to a self-test, self-tests also help to bound the computational power of entanglementassisted MIP protocols [10, 14, 24].

The case of self-testing of the EPR pair is fully understood. The techniques for this case were first introduced in [19], then improved in [3]. Selftesting of tensor products of maximally entangled qubits were proved in [22, 23], with the latter being the one with the smallest question and answer sets. Self-testing of a general bipartite entangled states with local dimension $d$ was proved in [8], which uses four questions but each question had $d$ answers. The number of questions was later reduced to two in [27], but the number of answers was still $d$.

Our work aims at minimizing the sizes of the question and answer sets of a correlation that can self-test a maximally entangled state with large local dimension. For comparison, all the correlations used in the results listed above have either the number of questions or answers dependent on the local dimension of the entangled state. Our progress is summarized in the following theorem.

Theorem 1. Let $r \in\{2,3,5\}$ and let $D$ denote the infinite set of all odd primes $p$ whose smallest primitive root is $r$. Then for any $p \in D$, the following maximally entangled state of local dimension $p-1$,

$$
|\tilde{\psi}\rangle=\frac{1}{\sqrt{p-1}} \sum_{j=1}^{p-1}|j\rangle|p-j\rangle,
$$

can be self-tested with constant-sized question and answer sets. 


\section{Proof overview}

To prove Theorem 1, we construct a bipartite quantum correlation $P_{p, r}$. We denote the two parties by Alice and Bob. Alice and Bob's question sets are of size $\Theta(r)$ and their answer sets are constant-sized for each odd prime number $p$ with smallest primitive root $r$. We say the size of the question set is of order $\Theta(r)$ to mean that there exist constants $c$ and $d$ such that the size of the question set is $c r+d$, and we say that $r$ is a primitive root of $p$ if $r$ is a multiplicative generator of the group $\mathbb{Z}_{p}^{*}$. Note that when $r \in\{2,3,5\}$ the size of neither the question set nor the answer set of $P_{p, r}$ depends on $p$. We can view $P_{p, r}$ in a matrix where each entry in the matrix is labelled by Alice's and Bob's question-answer pair, so the size of $P_{p, r}$ is the size of this matrix, which equals the product of the number of Alice's question-answer pairs and the number of Bob's question answer pairs. Therefore, the size of $P_{p, r}$ is of order $\Theta\left(r^{2}\right)$. We prove $P_{p, r}$ self-tests a maximally entangled state of dimension $(p-1)$.

The correlation $P_{p, r}$ contains a perfect correlation associated with a binary linear system. To induce such a correlation, Alice is given a random equation from a linear system and she should give an assignment to each variable of the equation. Bob is asked to assign a value to a random variable of the chosen equation. The correlation is perfect if Alice's assignment satisfies the equation and Bob's assignment to the variable matches Alice's assignment. A widely-used and thoroughlystudied example is the perfect correlation of the following linear system

$$
\begin{array}{ll}
x_{1}+x_{2}+x_{3}=0 & x_{4}+x_{5}+x_{6}=0 \\
x_{7}+x_{8}+x_{9}=0 & x_{1}+x_{4}+x_{7}=0 \\
x_{2}+x_{5}+x_{8}=1 & x_{3}+x_{6}+x_{9}=0 .
\end{array}
$$

The game version of this linear system is know as the Magic square game [2, 20]. Using two copies of $|E P R\rangle$, the perfect correlation can be induced. It has been shown that if a strategy can induce this correlation, the shared state must be $|E P R\rangle^{\otimes 2}$ up to some local isometry [29]. The key observation that leads to the self-testing proof is that an inducing strategy must contain binary observables $X$ and $Z$ that satisfy the anticommutation relation

$$
Z X Z=-X
$$

Intuitively, we can think of this perfect correlation as a way to enforce the anti-commutation relation [7].

We design a linear system $\hat{A} x=0$ such that the perfect correlation $P_{\hat{A}}$ associated with the linear system can enforce the relation

$$
U O U^{\dagger}=O^{r}
$$

for unitaries $U$ and $O$. The inspiration comes from Slofstra's seminal work [28], where he outlined a new way to design binary linear system such that the associated perfect correlation can enforce conjugacy relations of the form $X Y X^{\dagger}=$ $Z$ for unitaries $X, Y$ and $Z$. Following Slofstra's design, the numbers of equations and variables of $\hat{A} x=0$ are of order $\Theta(r)$.

The reason that we choose Equation (1) to be the relation enforced by $P_{\hat{A}}$ is the following. Inducing $P_{\hat{A}}$ guarantees that the strategy contains unitary operators $U$ and $O$ satisfying Equation (1). Moreover, if we can certify that the unitary $O$ has the eigenvalue $\omega_{p}:=e^{i 2 \pi / p}$, Equation (1) automatically guarantees that the spectrum of $O$ contains $\left\{\omega_{p}^{j} \mid 1 \leq j \leq p-1\right\}$. It also guarantees that the eigenspace of each distinct eigenvalue has the same dimension.

In order to certify an eigenvalue of $O$, we use the optimal correlation $P_{\mu}$ that achieves the maximal violation of the $\cot (\mu)$-weighted $\mathrm{CHSH}$ inequality proposed in $[1,16]$ as a variation of the CHSH inequality [5]

$$
\begin{aligned}
I_{\cot (\mu)} & =\cot (\mu)\left\langle M_{0} N_{0}+M_{0} N_{1}\right\rangle+\left\langle M_{1} N_{0}-M_{1} N_{1}\right\rangle \\
& \leq 2|\cot (\mu)|,
\end{aligned}
$$

for binary observables $M_{x}, N_{y}$ with $x, y \in\{0,1\}$. It has been proved that this correlation can selftest $|E P R\rangle[15]$. We further prove that in an inducing strategy, the product of Bob's observables has eigenvalues $e^{i 2 \mu}$ and $e^{-i 2 \mu}$.

Therefore, the full correlation $P_{p, r}$ is induced by an ideal strategy that can induce $P_{\hat{A}}$ and $P_{-\pi / p}$ for some odd prime $p$ whose primitive root is $r$. We can use $P_{-\pi / p}$ to certify that the unitary $O$ has eigenvalues $\omega_{p}$ and $\omega_{p}^{-1}$. Combining this observation with the fact that $O$ satisfies the relation Equation (1), we can prove that the correlation $P_{p, r}$ self-tests the state $|\tilde{\psi}\rangle$ defined by

$$
|\tilde{\psi}\rangle=\frac{1}{\sqrt{p-1}} \sum_{j=1}^{p-1}|j\rangle|p-j\rangle .
$$


More precisely, we prove that if some quantum strategy using a shared state $|\psi\rangle$ induces $P_{p, r}$, then there exist a local isometry $\Phi_{A} \otimes \Phi_{B}$ and a state $|j u n k\rangle$ such that

$$
\Phi_{A} \otimes \Phi_{B}(|\psi\rangle)=|j u n k\rangle \otimes|\tilde{\psi}\rangle .
$$

Note that the isometry $\Phi_{A} \otimes \Phi_{B}$ only involves local operations on Alice's and Bob's sides. The isometry $\Phi_{A} \otimes \Phi_{B}$ captures the essence of the generalized swap-isometry proposed in [30], but it is constructed in a different way.

The last step of proving Theorem 1 involves a number theory result. It has been shown that there exists $r \in\{2,3,5\}$ such that there are infinitely many primes whose smallest primitive is $r$ [13, 21]. The set $D$ in the statement of Theorem 1 is the set of all such primes and we can prove Theorem 1 by applying the self-testing result of $P_{p, r}$ to all $p \in D$.

\section{Structure of the paper}

We start with notations and background information in Section 1. We present a correlation that can enforce the relation $U O U^{\dagger}=O^{r}$ in Section 2 and present an extension of $P_{\mu}$ in Section 3. Then we define the correlation $P_{p, r}$ and prove its selftesting property in Section 4. Conclusions and open problems are discussed in Section 5.

\section{Preliminaries}

In this section, we introduce our notations and bipartite quantum correlations. The EPR pair is denoted by

$$
|E P R\rangle=\frac{1}{\sqrt{2}}(|00\rangle+|11\rangle) .
$$

The $d$-th root of unity is denoted by $\omega_{d}:=e^{2 \pi i / d}$. The set $\{0,1, \ldots n-1\}$ is denoted by $[n]$.

When we write operator relation with respect to a state, we use the following style. Let $\mathcal{H}_{A}$ and $\mathcal{H}_{B}$ be two Hilbert spaces. When it is clear from the context that unitaries $U_{A}$ and $U_{B}$ act on $\mathcal{H}_{A}$ and $\mathcal{H}_{B}$ respectively, we write $U_{A} \otimes U_{B}|\psi\rangle$ as $U_{A} U_{B}|\psi\rangle, U_{A} \otimes \mathbb{I}|\psi\rangle$ as $U_{A}|\psi\rangle$, and $\mathbb{I} \otimes U_{B}|\psi\rangle$ as $U_{B}|\psi\rangle$ for some $|\psi\rangle \in \mathcal{H}_{A} \otimes \mathcal{H}_{B}$.

To introduce bipartite quantum correlations, we consider a nonlocal scenario with two players, Alice and Bob. Each of them is requested to give an answer for some question. Alice's question is chosen from the set $\left[n_{A}\right]$ and her answer should be from the set $\left[m_{A}\right]$. Bob's question is chosen from the set $\left[n_{B}\right]$ and his answer should be from the set $\left[m_{B}\right]$. Therefore, a nonlocal scenario is described by the tuple $\left(\left[n_{A}\right],\left[n_{B}\right],\left[m_{A}\right],\left[m_{B}\right]\right)$. A bipartite correlation of a nonlocal scenario $\left(\left[n_{A}\right],\left[n_{B}\right],\left[m_{A}\right],\left[m_{B}\right]\right)$ is a function $P:\left[n_{A}\right] \times\left[n_{B}\right] \times\left[m_{A}\right] \times\left[m_{B}\right] \rightarrow \mathbb{R}_{\geq 0}:$ $(i, j, k, l) \mapsto P(k, l \mid i, j)$ where $P(k, l \mid i, j)$ is the probability for Alice to answer $k$ and Bob to answer $l$ when the question to Alice is $i$ and to Bob is $j$. The size of the correlation $P$ is $n_{A} n_{B} m_{A} m_{B}$.

Recall that a set of self-adjoint operators on $\mathcal{H},\left\{P_{j} \mid j \in[n]\right\}$, is a projective measurement if $P_{i}^{2}=P_{i}$ for all $i \in[n], P_{i} P_{j}=0$ for all $i \neq j$, and $\sum_{j \in[n]} P_{j}=\mathbb{I}_{\mathcal{H}}$. Each $P_{i}$ is called a projector. A binary observable on a Hilbert space $\mathcal{H}$ is a self-adjoint operator $M$ such that $M^{2}=\mathbb{I}_{\mathcal{H}}$.

A quantum projective measurement strategy for a nonlocal scenario $\left(\left[n_{A}\right],\left[n_{B}\right],\left[m_{A}\right],\left[m_{B}\right]\right)$ is a tuple

$$
\begin{array}{r}
\left(|\psi\rangle \in \mathcal{H}_{A} \otimes \mathcal{H}_{B},\left\{\left\{P_{i}^{(k)} \mid k \in\left[m_{A}\right]\right\} \mid i \in\left[n_{A}\right]\right\},\right. \\
\left.\left\{\left\{Q_{j}^{(l)} \mid l \in\left[m_{B}\right]\right\} \mid j \in\left[n_{B}\right]\right\}\right),
\end{array}
$$

where $\mathcal{H}_{A}$ and $\mathcal{H}_{B}$ are Hilbert spaces of arbitrary dimension, $\left\{\left\{P_{i}^{(k)} \mid k \in\left[m_{A}\right]\right\} \mid i \in\left[n_{A}\right]\right\}$ and $\left\{\left\{Q_{j}^{(l)} \mid l \in\left[m_{B}\right]\right\} \mid j \in\left[n_{B}\right]\right\}$ are two sets of projective measurements on $\mathcal{H}_{A}$ and $\mathcal{H}_{B}$ respectively. Note that the tensor product structure indicates that the two parties cannot communicate with each other, which is the reason why we say such scenario is nonlocal. This quantum strategy induces the bipartite quantum correlation

$$
P(k, l \mid i, j)=\left\langle\psi\left|P_{i}^{(k)} \otimes Q_{j}^{(l)}\right| \psi\right\rangle,
$$

for each $(i, j, k, l) \in\left[n_{A}\right] \times\left[n_{B}\right] \times\left[m_{A}\right] \times\left[m_{B}\right]$.

Next we introduce a type of correlations associated with a binary linear system. Let $A x=0$ be an $m \times n$ binary linear system, where each row of $A$ has $\kappa$ nonzero entries and each entry of $A$ is from $\mathbb{Z}_{2}$. For each $i \in[m]$, we define

$$
\begin{aligned}
& I_{i}=\{j \in[n] \mid A(i, j)=1\} \\
& S_{i}=\left\{x \in \mathbb{Z}_{2}^{I_{i}} \cong \mathbb{Z}_{2}^{\kappa} \mid \sum_{j \in I_{i}} x(j) \equiv 0 \quad(\bmod 2)\right\}
\end{aligned}
$$

where the isomorphism between $\mathbb{Z}_{2}^{I_{i}}$ and $\mathbb{Z}_{2}^{\kappa}$ is implicit. 
A correlation $P:[m] \times[n] \times \mathbb{Z}_{2}^{\kappa} \times \mathbb{Z}_{2} \rightarrow \mathbb{R}$ is a perfect correlation of $A x=0$ if

$$
\sum_{x, y: x \in S_{i}, x(j)=y} P(x, y \mid i, j)=1
$$

for all $i \in[m]$ and $j \in I_{i}$. The implication of a perfect correlation of $A x=0$ is summarized in the following lemma.

Lemma 1.1. For an $m$-by-n binary linear system $A x=0$ where each row of $A$ has $\kappa$ nonzero entries, if a quantum strategy

$$
\begin{aligned}
& \left(|\psi\rangle \in \mathcal{H}_{A} \otimes \mathcal{H}_{B},\right. \\
& \\
& \left\{\left\{P_{i}^{(x)} \mid x \in \mathbb{Z}_{2}^{\kappa}\right\} \mid i \in[m]\right\}, \\
& \left.\left\{\left\{Q_{j}^{(y)} \mid y \in \mathbb{Z}_{2}\right\} \mid j \in[n]\right\}\right)
\end{aligned}
$$

induces a perfect correlation of $A x=0$, then there exist a set of binary observables $\left\{M_{j} \mid j \in[n]\right\}$ on $\mathcal{H}_{A}$ and a set of binary observables $\left\{N_{j} \mid j \in[n]\right\}$ on $\mathcal{H}_{B}$ such that

$$
M_{j} N_{j}|\psi\rangle=|\psi\rangle
$$

for all $j \in[n]$,

$$
\prod_{j \in I_{i}} M_{j}|\psi\rangle=\prod_{j \in I_{i}} N_{j}|\psi\rangle=|\psi\rangle
$$

for all $i \in[m]$, and

$$
\left(M_{j} M_{k}\right)|\psi\rangle=\left(M_{k} M_{j}\right)|\psi\rangle
$$

for all $i \in[m]$ and $j, k \in I_{i}$.

The proof of the lemma can be found in the proof of Theorem 4 of [6] and in Section 3 of [28] so we omit it here.

Another correlation that we are interested in is the correlation that gives the maximal violation of the $\cot (\mu)$-weighted $\mathrm{CHSH}$ inequality defined in Equation (2).

We first give the inducing strategy of this correlation. Let $\mu \in[-\pi, \pi)$. Define

$$
\begin{array}{lll}
\tilde{P}_{0}^{(0)} & =|0\rangle\langle 0|, & \tilde{P}_{0}^{(1)}=|1\rangle\langle 1|, \\
\tilde{P}_{1}^{(0)}=\frac{1}{2}(|0\rangle+|1\rangle)(\langle 0|+\langle 1|), & \tilde{P}_{1}^{(1)}=\mathbb{I}-\tilde{P}_{1}^{(0)},
\end{array}
$$

and

$$
\begin{aligned}
\tilde{Q}_{0}^{(0)}= & \left(\cos \left(\frac{\mu}{2}\right)|0\rangle+\sin \left(\frac{\mu}{2}\right)|1\rangle\right) \\
& \times\left(\cos \left(\frac{\mu}{2}\right)\langle 0|+\sin \left(\frac{\mu}{2}\right)\langle 1|\right), \\
\tilde{Q}_{0}^{(1)}= & \mathbb{I}-\tilde{Q}_{0}^{(0)}, \\
\tilde{Q}_{1}^{(0)}= & \left(\cos \left(\frac{\mu}{2}\right)|0\rangle-\sin \left(\frac{\mu}{2}\right)|1\rangle\right) \\
& \times\left(\cos \left(\frac{\mu}{2}\right)\langle 0|-\sin \left(\frac{\mu}{2}\right)\langle 1|\right), \\
\tilde{Q}_{1}^{(1)}= & \mathbb{I}-\tilde{Q}_{1}^{(0)} .
\end{aligned}
$$

Definition 1.2. The correlation $P_{\mu}:[2] \times[2] \times$ $[2] \times[2] \rightarrow \mathbb{R}$ is induced by the strategy

$$
\begin{array}{r}
\left(|E P R\rangle,\left\{\left\{\tilde{P}_{x}^{(a)} \mid a \in[2]\right\} \mid x \in[2]\right\},\right. \\
\left.\left\{\left\{\tilde{Q}_{y}^{(b)} \mid b \in[2]\right\} \mid y \in[2]\right\}\right),
\end{array}
$$

such that $P_{\mu}(a, b \mid x, y)=\left\langle E P R\left|P_{x}^{(a)} Q_{y}^{(b)}\right| E P R\right\rangle$.

The correlation $P_{\mu}$ is known as the optimal correlation of the $\cot (\mu)$-weighted $\mathrm{CHSH}$ inequality. The self-testing property of $P_{\mu}$ is summarized in the following Lemma, which was first proved in [15, Proposition A.3].

Lemma 1.3. For $\mu \in[-\pi, \pi)$, if a quantum strategy $\left(|\psi\rangle,\left\{\left\{P_{x}^{(a)} \mid a \in[2]\right\} \mid x \in[2]\right\},\left\{\left\{Q_{y}^{(b)} \mid b \in\right.\right.\right.$ $[2]\} \mid y \in[2]\})$ induces $P_{\mu}$, then there exist a local isometry $\Phi=\Phi_{A} \otimes \Phi_{B}$ and an auxiliary state $\mid j$ unk $\rangle$ such that

$\Phi\left(P_{x}^{(a)} \otimes Q_{y}^{(b)}|\psi\rangle\right)=|j u n k\rangle \otimes\left(\tilde{P}_{x}^{(a)} \otimes \tilde{Q}_{y}^{(b)}\right)|E P R\rangle$ for $x, y \in\{-1,0,1\}$ where the subscript -1 refers to the identity operator and $\tilde{P}_{x}^{(a)}, \tilde{Q}_{y}^{(b)}$ for $x, y, a, b \in\{0,1\}$ are defined above.

Instead of giving the full proof, we list some key relations of this proof that will be reused later. From the projective measurements of the strategy, we get binary observables $P_{x}:=P_{x}^{(0)}-P_{x}^{(1)}$ and $Q_{y}:=Q_{y}^{(0)}-Q_{y}^{(1)}$ for $x, y \in[2]$. Define

$$
\begin{array}{lll}
Z_{A}:=P_{0}, & X_{A}:=P_{1}, \\
Z_{B}:=\frac{Q_{0}+Q_{1}}{2 \cos \mu}, & X_{B}:=\frac{Q_{0}-Q_{1}}{2 \sin \mu},
\end{array}
$$

then

$$
\begin{aligned}
& Z_{A}|\psi\rangle=Z_{B}|\psi\rangle, \\
& X_{A}|\psi\rangle=X_{B}|\psi\rangle, \\
& X_{A}\left(\mathbb{I}+Z_{B}\right)|\psi\rangle=X_{B}\left(\mathbb{I}-Z_{A}\right)|\psi\rangle, \\
& Z_{A}\left(\mathbb{I}+X_{B}\right)|\psi\rangle=Z_{B}\left(\mathbb{I}-X_{A}\right)|\psi\rangle, \\
& Z_{A} X_{A}|\psi\rangle=-X_{A} Z_{A}|\psi\rangle, \\
& X_{A} Z_{A}|\psi\rangle=-X_{B} Z_{B}|\psi\rangle .
\end{aligned}
$$




\section{Enforcing $U O U^{-1}=O^{r}$}

In this section, we show how to construct a binary linear system such that the perfect correlation associated with it can enforce the relation $U O U^{-1}=O^{r}$. Since the construction relies heavily on group presentations, we give the group theory background first. For more contexts, please refer to [26].

\subsection{Group theory background}

Let $S$ be a set of letters. We denote by $\mathcal{F}(S)$ the free group generated by $S$, which consists of all finite words made from $\left\{s, s^{-1} \mid s \in S\right\}$ such that no $s s^{-1}$ or $s^{-1} s$ appears as a substring for any $s$, where $s^{-1}$ denotes the inverse of $s$. The group law is given by concatenation and cancellation.

Definition 2.1 (Group presentation). Given a set $S$, let $\mathcal{F}(S)$ be the free group generated by $S$ and let $R$ be a subset of $\mathcal{F}(S)$. Then $\langle S: R\rangle$ denotes the quotient of $\mathcal{F}(S)$ by the normal subgroup generated by $R$. If a group $G$ is isomorphic to $\langle S: R\rangle$, then we say $G$ has a presentation $\langle S: R\rangle$.

If both sets $S$ and $R$ are finite and a group $G$ is defined by $\langle S: R\rangle$, then we say the group $G=\langle S: R\rangle$ is finitely-presented. The elements of $S$ are the generators and the elements of $R$ are the relations. A relation $r \in R$ is written as $r=e$ to convey its significance in the quotient group $G$. There are two types of relations that we will work with. Considering a subset of generators $\left\{s_{j} \mid 1 \leq\right.$ $j \leq n\} \subseteq S$, the relations of the form $\Pi_{j=1}^{n} s_{j}=e$, where $e$ is the identity element, are called linear relations; and the relations of the form $s_{i} s_{j} s_{i}^{-1}=$ $s_{k}$ for some $i \neq j$ are called conjugacy relations.

There are two special types of groups that we will work with in this section. We give the definitions below.

Definition 2.2 (Conjugacy group). Let $C \subseteq$ $[n] \times[n] \times[n]$, and

$$
\begin{aligned}
G=\left\langle\left\{s_{i} \mid i \in[n]\right\}:\right. & \left\{s_{i}^{2}=e \mid i \in[n]\right\} \cup \\
& \left.\left\{s_{i} s_{j} s_{i}=s_{k} \mid(i, j, k) \in C\right\}\right\rangle .
\end{aligned}
$$

We say a group is a conjugacy group if it has a presentation of this form.

A conjugacy group is a special case of what Slofstra defined as a linear-plus-conjugacy group (Definition 26 of [28]), since a conjugacy group does not have linear relations. The other type of group is directly related to a linear system.

Definition 2.3 (Solution group). Let $A x=0$ be an $m \times n$ binary linear system. The solution group of this system is the group

$$
\begin{aligned}
\Gamma(A):=\langle & \left\{x_{i} \mid i \in[n]\right\}:\left\{x_{j}^{2}=e \mid j \in[n]\right\} \\
& \cup\left\{\prod_{j \in I_{i}} x_{j}=e \mid i \in[m]\right\} \\
& \left.\cup\left\{x_{l} x_{k}=x_{k} x_{l} \mid k, l \in I_{i} \text { for some } i \in[m]\right\}\right\rangle .
\end{aligned}
$$

A representation of a group $G$ on a finitedimensional Hilbert space $\mathcal{H}$ is a group homomorphism from $G$ to the group of unitary operators on $\mathcal{H}$, denoted by $\mathcal{U}(\mathcal{H})$. The first reason that we study solution groups is that a representation of a solution group gives us a quantum strategy that induces a perfect correlation of the linear system [28, Theorem 18]. The second reason is that, given a solution group, we can construct a binary linear system by converting a relation of the form $x_{i} x_{j} x_{k}=e$ into a linear equation $x_{i}+x_{j}+x_{k}=0$. In the next subsection, we are going to construct a solution group first and then extract the binary linear system from it.

Lastly, for groups $G$ and $K$, an embedding of $G$ into $K$ is an injective group homomorphism $\phi: G \rightarrow K$. The relation between conjugacy groups and solution groups is that any conjugacy group can be embedded in a solution group, as proved in [28, Proposition 27].

\subsection{Constructing the linear system}

This subsection is devoted to proving the following proposition.

Proposition 2.4. Let $r \geq 2$ be a positive integer. There exists a binary linear system $\hat{A} x=0$ such that the following holds. If a quantum strategy $S=\left(|\psi\rangle \in \mathcal{H}_{A} \otimes \mathcal{H}_{B},\left\{\left\{P_{x}^{(a)}\right\}\right\},\left\{\left\{Q_{y}^{(b)}\right\}\right\}\right)$ induces a perfect correlation of $\hat{A} x=0$, then there exist binary observables $\left\{M_{u_{1}}, M_{u_{2}}, M_{o_{1}}, M_{o_{2}}\right\}$ on $\mathcal{H}_{A}$ and $\left\{N_{u_{1}}, N_{u_{2}}, N_{o_{1}}, N_{o_{2}}\right\}$ on $\mathcal{H}_{B}$ such that

$$
\begin{aligned}
& M_{u_{1}} M_{u_{2}}\left(M_{o_{1}} M_{o_{2}}\right) M_{u_{2}} M_{u_{1}}|\psi\rangle=\left(M_{o_{1}} M_{o_{2}}\right)^{r}|\psi\rangle \\
& N_{u_{1}} N_{u_{2}}\left(N_{o_{1}} N_{o_{2}}\right) N_{u_{2}} N_{u_{1}}|\psi\rangle=\left(N_{o_{1}} N_{o_{2}}\right)^{r}|\psi\rangle .
\end{aligned}
$$

Note that in the statement of Proposition $2.4 r$ can be any positive integer greater than 1 , and it doesn't have to be from the set $\{2,3,5\}$. To prove Proposition 2.4, we need the following lemma to 
combine operator relations with respect to the same shared state.

Lemma 2.5 (Lemma 7 of [4]). Let $|\psi\rangle \in \mathcal{H}$ be a quantum state. Suppose there exist unitaries $\{V\} \cup\left\{V_{i} \mid i \in[k]\right\} \cup\left\{M_{i} \mid i \in[n]\right\}$ on $\mathcal{H}$ commuting with $\left\{N_{i} \mid i \in[n]\right\}$ on $\mathcal{H}$ such that

$$
M_{i}|\psi\rangle=N_{i}|\psi\rangle
$$

for each $i \in[n]$, and

$$
V|\psi\rangle=\prod_{i \in[k]} V_{i}|\psi\rangle .
$$

Then,

$$
V \prod_{i \in[n]} M_{i}|\psi\rangle=\left(\prod_{i \in[k]} V_{i}\right)\left(\prod_{i \in[n]} M_{i}\right)|\psi\rangle .
$$

Proof of Proposition 2.4. The linear system $\hat{A} x=0$ is constructed from a solution group, wherein the following group is embedded. For $r \geq 2$, define

$$
G:=\left\langle u, o: u o u^{-1}=o^{r}\right\rangle .
$$

By [28, Proposition 33], $G$ can be embedded into a conjugacy group $G_{c}=\left\langle S_{c}: R_{c}\right\rangle$ where $S_{c}$ contains $\left\{u_{1}, u_{2}, o_{1}, o_{2}\right\}$ and $u_{1}^{2}=u_{2}^{2}=o_{1}^{2}=o_{2}^{2}=e$. We also know that the embedding $\phi: G \rightarrow G_{c}$ maps $u$ to $u_{1} u_{2}$ and $o$ to $o_{1} o_{2}$. In other words, in $G_{c}$

$$
u_{1} u_{2}\left(o_{1} o_{2}\right) u_{2} u_{1}=\left(o_{1} o_{2}\right)^{r} .
$$

By [28, Proposition 27], $G_{c}$ can be embedded into a solution group $\Gamma(\hat{A}):=\left\langle S_{\Gamma}, R_{\Gamma}\right\rangle$. Moreover, $\left\{u_{1}, u_{2}, o_{1}, o_{2}\right\} \subseteq S_{\Gamma}$ and the embed$\operatorname{ding} \phi^{\prime}: G_{c} \rightarrow \Gamma(\hat{A})$ maps $s$ to $s$ for each $s \in\left\{u_{1}, u_{2}, o_{1}, o_{2}\right\}$. Therefore, $G$ is embedded in $\Gamma(\hat{A})$ and we get the binary linear system $\hat{A} x=0$.

Since $G_{c}$ is embedded in $\Gamma(\hat{A})$, we know that the relation in Equation (13) can be reconstructed by substituting in relations in $R_{\Gamma}$. Then, the statement of the proposition follows from Lemmas 1.1 and 2.5.

Following [28, Remark 30], we can get that $\hat{A} x=0$ has $n(r):=16 s+75$ variables and $m(r):=14 s+62$ equations, where each equation has 3 nonzero variables. We assume that in this system $x_{0}$ corresponds to $o_{1}$ and $x_{1}$ corresponds to $\mathrm{O}_{2}$.

Next we show that there exists a quantum strategy that can induce a perfect correlation of
$\hat{A} x=0$. The correlation is denoted by $P_{\hat{A}}$ and the strategy is denoted by $S_{\hat{A}}$, which is based on a representation of $\Gamma(\hat{A})$.

We first give a representation of $G_{c}$. Let $p$ be an odd prime number such that $r$ is a primitive root of $p$. Note that here we don't require that $r$ is the smallest primitive root of $p$. Let $\mathbb{C}^{p-1}=$ $\operatorname{span}(\{|j\rangle \mid 1 \leq j \leq p-1\})$. A second basis of $\mathbb{C}^{p-1}$ is $\left\{\left|x_{j}\right\rangle \mid 1 \leq j \leq p-1\right\}$, where

$$
\begin{aligned}
& \left|x_{j}\right\rangle=-\frac{1}{\sqrt{2}}(|j\rangle+i|p-j\rangle), \\
& \left|x_{p-j}\right\rangle=\frac{-\omega_{2 p}^{j}}{\sqrt{2}}(|j\rangle-i|p-j\rangle)
\end{aligned}
$$

for $1 \leq j \leq \frac{p-1}{2}$. Note that another form of this basis is $\left\{\left|x_{r^{j}}\right\rangle \mid j \in[p-1]\right\}$, where the subscript $r^{j}$ is taken modulo $p$ implicitly. Based on the second basis, we define a third basis of $\mathbb{C}^{p-1},\left\{\left|u_{k}\right\rangle \mid k \in\right.$ $[p-1]\}$ defined by

$$
\left|u_{k}\right\rangle=\frac{1}{\sqrt{p-1}} \sum_{j=0}^{p-2} \omega_{p-1}^{j k}\left|x_{r^{j}}\right\rangle .
$$

On $\mathbb{C}^{p-1}$, we define

$$
\begin{aligned}
O_{1} & =\sum_{j=1}^{(p-1) / 2} \omega_{p}^{j}\left|x_{j}\right\rangle\left\langle x_{p-j}\left|+\omega_{p}^{-j}\right| x_{p-j}\right\rangle\left\langle x_{j}\right|, \\
O_{2} & =\sum_{j=1}^{(p-1) / 2}\left|x_{j}\right\rangle\left\langle x_{p-j}|+| x_{p-j}\right\rangle\left\langle x_{j}\right|, \\
U_{1} & =\left|u_{0}\right\rangle\left\langle u_{0}|-| u_{(p-1) / 2}\right\rangle\left\langle u_{(p-1) / 2}\right| \\
& +\sum_{k=1}^{(p-3) / 2}\left(\omega_{p-1}^{k}\left|u_{k}\right\rangle\left\langle u_{p-1-k}\left|+\omega_{p-1}^{-k}\right| u_{p-1-k}\right\rangle\left\langle u_{k}\right|\right),
\end{aligned}
$$

$$
\begin{aligned}
U_{2} & =\left|u_{0}\right\rangle\left\langle u_{0}|+| u_{(p-1) / 2}\right\rangle\left\langle u_{(p-1) / 2}\right| \\
& +\sum_{k=1}^{(p-3) / 2}\left(\left|u_{p-1-k}\right\rangle\left\langle u_{k}|+| u_{k}\right\rangle\left\langle u_{p-1-k}\right|\right) .
\end{aligned}
$$

It can be checked that

$$
\begin{aligned}
& O_{1} O_{2}=\sum_{j \in[p-1]} \omega_{p}^{r^{j}}\left|x_{r^{j}}\right\rangle\left\langle x_{r^{j}}\right|, \\
& U_{1} U_{2}=\sum_{j \in[p-1]}\left|x_{r^{j-1}}\right\rangle\left\langle x_{r^{j}}\right|, \\
& U_{1} U_{2}\left(O_{1} O_{2}\right) U_{2} U_{1}=\left(O_{1} O_{2}\right)^{r} .
\end{aligned}
$$

Hence, we can follow the proof of [28, Proposition 33 to extend $\rho: G_{c} \rightarrow \mathcal{U}\left(\mathbb{C}^{p-1}\right): u_{1} \mapsto U_{1}, u_{2} \mapsto$ 
$U_{2}, o_{1} \mapsto O_{1}, o_{2} \mapsto O_{2}$ to a representation of $G_{c}$, still denoted by $\rho$. Then, following the proofs of [28, Proposition 27 and Lemma 29], $\rho$ can be extended to a representation of $\Gamma(\hat{A}), \rho^{\prime}: \Gamma(\hat{A}) \rightarrow$ $\mathcal{U}\left(\mathbb{C}^{p-1} \otimes \mathbb{C}^{2} \otimes \mathbb{C}^{2}\right)$. In particular, for any $s \in$ $\left\{u_{1}, u_{2}, o_{1}, o_{2}\right\}$

$$
\rho^{\prime}(s)=\rho(s) \otimes \mathbb{I}_{\mathbb{C}^{2}} \otimes \mathbb{I}_{\mathbb{C}^{2}} .
$$

Let

$$
|\tilde{\psi}\rangle:=\frac{1}{\sqrt{p-1}} \sum_{j=1}^{p-1}\left|x_{j}\right\rangle\left|x_{p-j}\right\rangle,
$$

and $\Pi_{s}^{(0)}, \Pi_{s}^{(1)}$ be the projectors onto the +1 and -1-eigenspaces of $\rho^{\prime}(s)$ for each $s \in S_{\Gamma}$. Then we can construct a strategy

$$
\begin{aligned}
S_{\hat{A}}= & \left(|\tilde{\psi}\rangle \otimes|E P R\rangle^{\otimes 2},\right. \\
& \left\{\left\{P_{i}^{(x)} \mid x \in \mathbb{Z}_{2}^{3}\right\} \mid i \in[m(r)]\right\}, \\
& \left.\left\{\left\{\Pi_{s}^{(y)} \mid y \in \mathbb{Z}_{2}\right\} \mid s \in S_{\Gamma}\right\}\right),
\end{aligned}
$$

where $P_{i}^{(x)}$ can be constructed from $\left\{\Pi_{s}^{(0)}, \Pi_{s}^{(1)} \mid s \in S_{\Gamma}\right\}$. Note that since the variables of $\hat{A}$ are in one-to-one correspondence with the generators in $S_{\Gamma}$, we label Bob's projectors by the corresponding generators.

Definition 2.6. The correlation $P_{\hat{A}}:[m(r)] \times$ $S_{\Gamma} \times \mathbb{Z}_{2}^{3} \times \mathbb{Z}_{2} \rightarrow R$ is defined by

$$
\begin{aligned}
& P_{\hat{A}}(x, y \mid i, s) \\
= & {\left[\left\langle\tilde { \psi } \left|\otimes\left\langle\left. E P R\right|^{\otimes 2}\right]\left(P_{i}^{(x)} \Pi_{s}^{(y)}\right)\left[|\tilde{\psi}\rangle \otimes|E P R\rangle^{\otimes 2}\right] .\right.\right.\right.}
\end{aligned}
$$

It can be checked that $P_{\hat{A}}$ is a perfect correlation of $\hat{A} x=0$.

\section{Extending the correlation $P_{\mu}$}

\subsection{Revisiting the self-testing property of $P_{\mu}$}

In this section, we show how to use $P_{\mu}$ to argue the eigenvalue of an unknown unitary.

Proposition 3.1. For $\mu \in[-\pi, \pi)$, if a quantum strategy $\left(|\psi\rangle \in \mathcal{H}_{A} \otimes \mathcal{H}_{B},\left\{\left\{P_{x}^{(a)} \mid a \in[2]\right\} \mid x \in\right.\right.$ $\left.[2]\},\left\{\left\{Q_{y}^{(b)} \mid b \in[2]\right\} \mid y \in[2]\right\}\right)$ induces $P_{\mu}$, then there exist quantum states $\left|\psi_{1}\right\rangle,\left|\psi_{2}\right\rangle \in \mathcal{H}_{A} \otimes \mathcal{H}_{B}$ such that

$$
\begin{aligned}
& Q_{0} Q_{1}\left|\psi_{1}\right\rangle=e^{-i 2 \mu}\left|\psi_{1}\right\rangle, \\
& Q_{0} Q_{1}\left|\psi_{2}\right\rangle=e^{i 2 \mu}\left|\psi_{2}\right\rangle,
\end{aligned}
$$

where $Q_{y}:=Q_{y}^{(0)}-Q_{y}^{(1)}$ for $y \in[2]$.
Proof. Let $P_{x}=P_{x}^{(0)}-P_{x}^{(1)}$ for $x \in\{0,1\}$. The states in Proposition 3.1 are

$$
\begin{aligned}
& \left|\psi_{1}\right\rangle=\left(P_{0}^{(0)}+i P_{1} P_{0}^{(1)}\right)|\psi\rangle \\
& \left|\psi_{2}\right\rangle=\left(P_{0}^{(0)}-i P_{1} P_{0}^{(1)}\right)|\psi\rangle
\end{aligned}
$$

We first show that $\|\left|\psi_{1}\right\rangle \|=1$.

$$
\begin{aligned}
& \|\left|\psi_{1}\right\rangle \|^{2} \\
= & \langle\psi|\left(P_{0}^{(0)}+P_{0}^{(1)}-i P_{0}^{(1)} P_{1} P_{0}^{(0)}+i P_{0}^{(0)} P_{1} P_{0}^{(1)}|\psi\rangle\right. \\
= & 1-i\left\langle\psi\left|P_{0}^{(1)} P_{1} P_{0}^{(0)}-P_{0}^{(0)} P_{1} P_{0}^{(1)}\right| \psi\right\rangle .
\end{aligned}
$$

Recall that $Z_{A}=P_{0}, X_{A}=P_{1}, Z_{B}=\left(Q_{0}+\right.$ $\left.Q_{1}\right) / 2 \cos (\mu)$ and $X_{B}=\left(Q_{0}-Q_{1}\right) / 2 \sin (\mu)$. Using eqs. (7) and (11), we know

$$
\begin{aligned}
P_{1} P_{0}^{(0)}|\psi\rangle & =\frac{X_{A}\left(\mathbb{I}+Z_{A}\right)}{2}|\psi\rangle \\
& =\frac{X_{B}\left(\mathbb{I}-Z_{B}\right)}{2}|\psi\rangle \\
& =\frac{\left(\mathbb{I}+Z_{B}\right) X_{B}}{2}|\psi\rangle \\
P_{0}^{(1)}|\psi\rangle & =\frac{\mathbb{I}-Z_{A}}{2}|\psi\rangle \\
& =\frac{\mathbb{I}-Z_{B}}{2}|\psi\rangle,
\end{aligned}
$$

so $\left\langle\psi\left|P_{0}^{(1)} P_{1} P_{0}^{(0)}\right| \psi\right\rangle=0$. With similar reasoning, we get $\left\langle\psi\left|P_{0}^{(0)} P_{1} P_{0}^{(1)}\right| \psi\right\rangle=0$. Therefore, $\|\left|\psi_{1}\right\rangle \|=$ 1. The derivation of $\|\left|\psi_{2}\right\rangle \|=1$ is very similar, so we omit it here.

Next, we show $Q_{0} Q_{1}\left|\psi_{1}\right\rangle=e^{-i 2 \mu}\left|\psi_{1}\right\rangle$ and $Q_{0} Q_{1}\left|\psi_{2}\right\rangle=e^{i 2 \mu}\left|\psi_{2}\right\rangle$. From eq. (6), we get that

$$
\begin{aligned}
Z_{B} P_{0}^{(0)}|\psi\rangle & =\frac{Z_{B}\left(\mathbb{I}+Z_{A}\right)}{2}|\psi\rangle \\
& =\frac{Z_{B}+\mathbb{I}}{2}|\psi\rangle \\
& =\frac{\mathbb{I}+Z_{A}}{2}|\psi\rangle \\
& =P_{0}^{(0)}|\psi\rangle .
\end{aligned}
$$

With similar reasoning, we get

$$
Z_{B} P_{0}^{(1)}|\psi\rangle=-P_{0}^{(1)}|\psi\rangle .
$$

Substituting the expression of $Z_{B}$, we see that

$$
\begin{aligned}
& \left(Q_{0}+Q_{1}\right) P_{0}^{(0)}|\psi\rangle=2 \cos (\mu) P_{0}^{(0)}|\psi\rangle \\
& \left(Q_{0}+Q_{1}\right) P_{0}^{(1)}|\psi\rangle=-2 \cos (\mu) P_{0}^{(1)}|\psi\rangle
\end{aligned}
$$


From eqs. (6) to (8) and (10), we get that

$$
\begin{aligned}
& X_{B} P_{0}^{(0)}|\psi\rangle=X_{A} P_{0}^{(1)}|\psi\rangle, \\
& X_{B} P_{0}^{(1)}|\psi\rangle=X_{A} P_{0}^{(0)}|\psi\rangle .
\end{aligned}
$$

Substituting in the expression of $X_{B}$, we get that

$$
\begin{aligned}
& \left(Q_{0}-Q_{1}\right) P_{0}^{(0)}|\psi\rangle=2 \sin (\mu) P_{1} P_{0}^{(1)}|\psi\rangle, \\
& \left(Q_{0}-Q_{1}\right) P_{0}^{(1)}|\psi\rangle=2 \sin (\mu) P_{1} P_{0}^{(0)}|\psi\rangle .
\end{aligned}
$$

Simple calculation gives us that

$$
\begin{aligned}
Q_{0} P_{0}^{(0)}|\psi\rangle & =\left[\cos (\mu) P_{0}^{(0)}+\sin (\mu) P_{1} P_{0}^{(1)}\right]|\psi\rangle, \\
Q_{1} P_{0}^{(0)}|\psi\rangle & =\left[\cos (\mu) P_{0}^{(0)}-\sin (\mu) P_{1} P_{0}^{(1)}\right]|\psi\rangle, \\
Q_{0} P_{0}^{(1)}|\psi\rangle & =\left[-\cos (\mu) P_{0}^{(1)}+\sin (\mu) P_{1} P_{0}^{(0)}\right]|\psi\rangle, \\
Q_{1} P_{0}^{(1)}|\psi\rangle & =\left[-\cos (\mu) P_{0}^{(1)}-\sin (\mu) P_{1} P_{0}^{(0)}\right]|\psi\rangle .
\end{aligned}
$$

Then,

$Q_{0} Q_{1} P_{0}^{(0)}|\psi\rangle=\left[\cos (2 \mu) P_{0}^{(0)}+\sin (2 \mu) P_{1} P_{0}^{(1)}\right]|\psi\rangle$, $Q_{0} Q_{1} P_{1} P_{0}^{(1)}|\psi\rangle=\left[\cos (2 \mu) P_{1} P_{0}^{(1)}-\sin (2 \mu) P_{0}^{(0)}\right]|\psi\rangle$.

Finally, the fact that $\left|\psi_{1}\right\rangle$ and $\left|\psi_{2}\right\rangle$ are $e^{-2 i \mu}$ and $e^{2 i \mu}$ eigenvectors pf $Q_{0} Q_{1}$ follows Equations (22) and (23).

\subsection{The correlation $\hat{P}_{-\pi / p}$}

In the rest of the work, we fix $\mu=-\pi / p$ for some odd prime $p$. We will introduce a correlation that is extended from $P_{-\pi / p}$ and the correlation is denoted by $\hat{P}_{-\pi / p}$. We define $\hat{P}_{-\pi / p}:[5] \times[5] \times$ $[3] \times[3] \rightarrow \mathbb{R}$ by defining its inducing quantum strategy.

In $\mathbb{C}^{p-1}$, we define a subspace $V=$ $\operatorname{span}(\{|1\rangle,|p-1\rangle\})$ and we denote the projector onto $V$ by $\Pi_{V}$. For question $x=0$, let

$$
\bar{P}_{0}^{(a)}=\bar{Q}_{0}^{(a)}= \begin{cases}\Pi_{V} & \text { if } a=0 \\ \mathbb{I}-\Pi_{V} & \text { if } a=1 \\ 0 & \text { otherwise. }\end{cases}
$$

For questions $x=1,2$, let $\Lambda_{O_{x}}^{(0)}, \Lambda_{O_{x}}^{(1)}$ be the projectors onto the +1 - and -1-eigenspaces of $O_{x}$, where $O_{1}$ and $O_{2}$ are defined in Equations (16) and (17), and let

$$
\bar{P}_{x}^{(a)}=\bar{Q}_{x}^{(a)}= \begin{cases}\Lambda_{O_{x}}^{(0)} & \text { if } a=0 \\ \Lambda_{O_{x}}^{(1)} & \text { if } a=1 \\ 0 & \text { otherwise. }\end{cases}
$$

For question $x=3$, let

$$
\bar{P}_{3}^{(a)}=\bar{Q}_{3}^{(a)}= \begin{cases}|1\rangle\langle 1| & \text { if } a=0 \\ |p-1\rangle\langle p-1| & \text { if } a=1 \\ \mathbb{I}-\Pi_{V} & \text { otherwise. }\end{cases}
$$

For question $x=4$, let

$\bar{P}_{4}^{(a)}=\bar{Q}_{4}^{(a)}= \begin{cases}\left.\frac{(|1\rangle+|p-1\rangle)(\langle 1|+\langle p-1|}{2}\right) & \text { if } a=0 \\ \left.\frac{(|1\rangle-|p-1\rangle)(\langle 1|-\langle p-1|}{2}\right) & \text { if } a=1 \\ \mathbb{I}-\Pi_{V} & \text { otherwise. }\end{cases}$

Substituting Equations (14) and (15) into Equation (20), we get

$$
|\tilde{\psi}\rangle=\frac{1}{\sqrt{p-1}} \sum_{j=1}^{(p-1) / 2} \omega_{2 p}^{j}(|j\rangle|j\rangle+|p-j\rangle|p-j\rangle) .
$$

The inducing strategy is

$$
\begin{array}{r}
S_{-\pi / p}=\left(|\tilde{\psi}\rangle,\left\{\left\{\bar{P}_{x}^{(a)} \mid a \in[3]\right\} \mid x \in[5]\right\},\right. \\
\left.\left\{\left\{\bar{Q}_{y}^{(b)} \mid b \in[3]\right\} \mid y \in[5]\right\}\right) .
\end{array}
$$

Definition 3.2. The correlation $\hat{P}_{-\pi / p}:[5] \times$ $[5] \times[3] \times[3] \rightarrow \mathbb{R}$ is induced by $S_{-\pi / p}$ :

$$
\hat{P}_{-\pi / p}(a, b \mid x, y)=\left\langle\tilde{\psi}\left|\bar{P}_{x}^{(a)} \bar{Q}_{y}^{(b)}\right| \tilde{\psi}\right\rangle .
$$

As an analogue of Proposition 3.1, the implication of $\hat{P}_{-\pi / p}$ is summarized in the next proposition.

Proposition 3.3. Let $\left(|\psi\rangle \in \mathcal{H}_{A} \otimes\right.$ $\mathcal{H}_{B},\left\{\left\{P_{x}^{(a)} \mid a \in[3]\right\} \mid x \in[5]\right\},\left\{\left\{Q_{y}^{(b)} \mid b \in[3]\right\} \mid y \in\right.$ $[5]\})$ be a strategy that induces $\hat{P}_{-\pi / p}$, and let

$$
\left|\psi_{1}\right\rangle=\frac{1}{2}\left(P_{3}^{(0)}+i P_{4} P_{3}^{(1)}-i P_{4} P_{3}^{(0)}+P_{3}^{(1)}\right)|\psi\rangle,
$$

where $P_{4}=P_{4}^{(0)}-P_{4}^{(1)}$. Then, $\|\left|\psi_{1}\right\rangle \|^{2}=1 /(p-1)$ and

$$
\begin{aligned}
& P_{1} P_{2}\left|\psi_{1}\right\rangle=\omega_{p}^{-1}\left|\psi_{1}\right\rangle \\
& Q_{1} Q_{2}\left|\psi_{1}\right\rangle=\omega_{p}\left|\psi_{1}\right\rangle,
\end{aligned}
$$

where $P_{x}:=P_{x}^{(0)}-P_{x}^{(1)}$ and $Q_{y}:=Q_{y}^{(0)}-Q_{y}^{(1)}$ for $x, y \in\{1,2\}$. 
To help with the proof of this proposition, we first give some values of $\hat{P}_{-\pi / p}$.

$\hat{P}_{-\pi / p}(a, b \mid 0,0)= \begin{cases}2 /(p-1) & \text { if } a=b=0 \\ (p-3) /(p-1) & \text { if } a=b=1 \\ 0 & \text { otherwise. }\end{cases}$

$\hat{P}_{-\pi / p}(a, b \mid 3,3)= \begin{cases}1 /(p-1) & \text { if } a=b=0 \\ 1 /(p-1) & \text { if } a=b=1 \\ (p-3) /(p-1) & \text { if } a=b=2 \\ 0 & \text { otherwise. }\end{cases}$

$\hat{P}_{-\pi / p}(a, b \mid 0,3)=\left\{\begin{array}{l}1 /(p-1) \\ (p-3) /(p-1) \\ 0\end{array}\right.$

if $a=0, b \in$

if $a=1, b=2$

otherwise.

\begin{tabular}{|c|c|c|c|c|c|}
\hline \multicolumn{2}{|c|}{} & \multicolumn{2}{|c|}{$x=3$} & \multicolumn{2}{c|}{$x=4$} \\
\cline { 2 - 6 } & $a=0$ & $a=1$ & $a=0$ & $a=1$ \\
\hline \hline \multirow{2}{*}{$y=1$} & $b=0$ & $\frac{\cos ^{2}(\pi / 2 p)}{p-1}$ & $\frac{\sin ^{2}(\pi / 2 p)}{p-1}$ & $\frac{1-\sin (\pi / p)}{2(p-1)}$ & $\frac{1+\sin (\pi / p)}{2(p-1)}$ \\
\cline { 2 - 6 } & $b=1$ & $\frac{\sin ^{2}(\pi / 2 p)}{p-1}$ & $\frac{\cos ^{2}(\pi / 2 p)}{p-1}$ & $\frac{1+\sin (\pi / p)}{2(p-1)}$ & $\frac{1-\sin (\pi / p)}{2(p-1)}$ \\
\hline \multirow{2}{*}{$y=2$} & $b=0$ & $\frac{\cos ^{2}(\pi / 2 p)}{p-1}$ & $\frac{\sin ^{2}(\pi / 2 p)}{p-1}$ & $\frac{1+\sin (\pi / p)}{2(p-1)}$ & $\frac{1-\sin (\pi / p)}{2(p-1)}$ \\
\cline { 2 - 6 } & $b=1$ & $\frac{\sin ^{2}(\pi / 2 p)}{p-1}$ & $\frac{\cos ^{2}(\pi / 2 p)}{p-1}$ & $\frac{1-\sin (\pi / p)}{2(p-1)}$ & $\frac{1+\sin (\pi / p)}{2(p-1)}$ \\
\hline
\end{tabular}

Table 1: The correlation for $x \in\{3,4\}, y \in\{1,2\}$ and $a, b \in[2]$.

Proof. From the definition of $\hat{P}_{-\pi / p}$, it is easy to see that

$$
P_{x}^{(2)}|\psi\rangle=Q_{x}^{(2)}|\psi\rangle=0
$$

for $x, y \in[3]$. Then

$$
\begin{aligned}
P_{x}^{2}|\psi\rangle & =\left[P_{x}^{(0)}+P_{x}^{(1)}\right]|\psi\rangle+P_{x}^{(2)}|\psi\rangle \\
& =|\psi\rangle
\end{aligned}
$$

for $x \in\{1,2\}$. Similarly, we see that $Q_{y}^{2}|\psi\rangle=|\psi\rangle$ for $y \in\{1,2\}$.

Next, we will show

$$
\begin{aligned}
P_{0}^{(0)}|\psi\rangle & =\left(P_{3}^{(0)}+P_{3}^{(1)}\right)|\psi\rangle \\
& =\left(P_{4}^{(0)}+P_{4}^{(1)}\right)|\psi\rangle \\
& =Q_{0}^{(0)}|\psi\rangle \\
& =\left(Q_{3}^{(0)}+Q_{3}^{(1)}\right)|\psi\rangle \\
& =\left(Q_{4}^{(0)}+Q_{4}^{(1)}\right)|\psi\rangle \\
P_{0}^{(1)}|\psi\rangle & =P_{3}^{(2)}|\psi\rangle=P_{4}^{(2)}|\psi\rangle= \\
Q_{0}^{(1)}|\psi\rangle & =Q_{3}^{(2)}|\psi\rangle=Q_{4}^{(2)}|\psi\rangle,
\end{aligned}
$$

and

$$
\begin{array}{ll}
P_{3}^{(0)}|\psi\rangle=Q_{3}^{(0)}|\psi\rangle & P_{3}^{(1)}|\psi\rangle=Q_{3}^{(1)}|\psi\rangle \\
P_{4}^{(0)}|\psi\rangle=Q_{4}^{(0)}|\psi\rangle & P_{4}^{(1)}|\psi\rangle=Q_{4}^{(1)}|\psi\rangle .
\end{array}
$$

Instead of giving the full proof, we show $P_{0}^{(0)}|\psi\rangle=$ $Q_{0}^{(0)}|\psi\rangle$ to demonstrate the ideas. From the correlation, we know $\left\langle\psi\left|P_{0}^{(0)} Q_{0}^{(0)}\right| \psi\right\rangle=\left\langle\psi\left|P_{0}^{(0)}\right| \psi\right\rangle=$ $\left\langle\psi\left|Q_{0}^{(0)}\right| \psi\right\rangle=2 /(p-1)$. Then,

$$
\| P_{0}^{(0)}|\psi\rangle-Q_{0}^{(0)}|\psi\rangle \|^{2}
$$

$$
\begin{aligned}
& =\left\langle\psi\left|P_{0}^{(0)}\right| \psi\right\rangle+\left\langle\psi\left|Q_{0}^{(0)}\right| \psi\right\rangle-2\left\langle\psi\left|P_{0}^{(0)} Q_{0}^{(0)}\right| \psi\right\rangle \\
& =0
\end{aligned}
$$

which implies that $P_{0}^{(0)}|\psi\rangle=Q_{0}^{(0)}|\psi\rangle$.

Then, we can show that $\hat{P}_{-\pi / p}$ can be "reduced" to $P_{-\pi / p}$ by proving that

$$
\begin{array}{r}
S=\left(\frac{P_{0}^{(0)}|\psi\rangle}{\| P_{0}^{(0)}|\psi\rangle \|},\left\{\left\{P_{x}^{(0)}, P_{x}^{(1)}\right\} \mid x \in\{3,4\}\right\},\right. \\
\left.\left\{\left\{Q_{y}^{(0)}, Q_{y}^{(1)}\right\} \mid y \in\{1,2\}\right\}\right)
\end{array}
$$

can induce $P_{-\pi / p}$, and that

$$
\begin{array}{r}
S^{\prime}=\left(\frac{P_{0}^{(0)}|\psi\rangle}{\| P_{0}^{(0)}|\psi\rangle \|},\left\{\left\{P_{x}^{(0)}, P_{x}^{(1)}\right\} \mid x \in\{1,2\}\right\},\right. \\
\left.\left\{\left\{Q_{y}^{(0)}, Q_{y}^{(1)}\right\} \mid y \in\{3,4\}\right\}\right)
\end{array}
$$

can induce $P_{-\pi / p}$ with Alice and Bob's roles flipped. To prove $S$ can induce $P_{-\pi / p}$, we need to examine the terms of the form $\left\langle\psi\left|P_{0}^{(0)} P_{x}^{(a)} Q_{y}^{(b)} P_{0}^{(0)}\right| \psi\right\rangle$ for $x=3,4, y=1,2$ and $a, b=0,1$. We find that these terms relate to $\left\langle\psi\left|P_{x}^{(a)} Q_{y}^{(b)}\right| \psi\right\rangle$ by

$$
\begin{aligned}
& \left\langle\psi\left|P_{x}^{(a)} Q_{y}^{(b)}\right| \psi\right\rangle \\
= & \left\langle\psi\left|\left(P_{0}^{(0)}+P_{0}^{(1)}\right) P_{x}^{(a)} Q_{y}^{(b)}\left(P_{0}^{(0)}+P_{0}^{(1)}\right)\right| \psi\right\rangle \\
= & \left\langle\psi\left|P_{0}^{(0)} P_{x}^{(a)} Q_{y}^{(b)} P_{0}^{(0)}\right| \psi\right\rangle,
\end{aligned}
$$

where we use the facts that $P_{x}^{(a)} P_{0}^{(1)}|\psi\rangle=$ $P_{x}^{(a)} P_{x}^{(2)}|\psi\rangle=0$ for the relevant values of $(x, y, a, b)$. Therefore,

$$
\frac{\left\langle\psi\left|P_{0}^{(0)} P_{x}^{(a)} Q_{y}^{(b)} P_{0}^{(0)}\right| \psi\right\rangle}{\| P_{0}^{(0)}|\psi\rangle \|^{2}}=\frac{\left\langle\psi\left|P_{x}^{(a)} Q_{y}^{(b)}\right| \psi\right\rangle}{\| P_{0}^{(0)}|\psi\rangle \|^{2}},
$$

for the relevant values of $(x, y, a, b)$, and it is easy to verify that $S$ induces $P_{-\pi / p}$. The proof of $S^{\prime}$ 
induces $P_{-\pi / p}$ with Alice and Bob's roles flipped is similar, so we omit it here.

The state $\left|\psi_{1}\right\rangle$ can also be written as

$$
\left|\psi_{1}\right\rangle=\frac{1}{2}\left(\mathbb{I}-i P_{4}\right)\left(P_{3}^{(0)}+i P_{4} P_{3}^{(1)}\right)|\psi\rangle .
$$

The derivation of $\|\left|\psi_{1}\right\rangle \|$ is very similar to the corresponding part in the proof of Proposition 3.1, so we omit it here. Since $S$ can induce $P_{-\pi / p}$, by Proposition 3.1, we know that

$$
\begin{aligned}
& Q_{1} Q_{2}\left(P_{3}^{(0)}+i P_{4} P_{3}^{(1)}\right) P_{0}^{(0)}|\psi\rangle \\
= & \omega_{p}\left(P_{3}^{(0)}+i P_{4} P_{3}^{(1)}\right) P_{0}^{(0)}|\psi\rangle .
\end{aligned}
$$

On the other hand,

$$
\begin{aligned}
& \left(P_{3}^{(0)}+i P_{4} P_{3}^{(1)}\right) P_{0}^{(0)}|\psi\rangle \\
= & \left(P_{3}^{(0)}+i P_{4} P_{3}^{(1)}\right)\left(P_{3}^{(0)}+P_{3}^{(1)}\right)|\psi\rangle \\
= & \left(P_{3}^{(0)}+i P_{4} P_{3}^{(1)}\right)|\psi\rangle .
\end{aligned}
$$

Hence, using the fact that $Q_{1} Q_{2}$ commutes with $\left(\mathbb{I}-i P_{4}\right)$, we know

$$
Q_{1} Q_{2}\left|\psi_{1}\right\rangle=\omega_{p}\left|\psi_{1}\right\rangle \text {. }
$$

What remains to be proved is $P_{1} P_{2}\left|\psi_{1}\right\rangle=$ $\omega_{p}^{-1}\left|\psi_{1}\right\rangle$. In order to prove it, we need another form of $\left|\psi_{1}\right\rangle$, which is

$$
\begin{aligned}
\left|\psi_{1}\right\rangle & =\frac{1}{2}\left(Q_{3}^{(0)}-i Q_{4} Q_{3}^{(1)}+i Q_{4} Q_{3}^{(0)}+Q_{3}^{(1)}\right)|\psi\rangle . \\
& =\left(\mathbb{I}+i Q_{4}\right)\left(Q_{3}^{(0)}-i Q_{4} Q_{3}^{(1)}\right)|\psi\rangle,
\end{aligned}
$$

where $Q_{4}:=Q_{4}^{(0)}-Q_{4}^{(1)}$. Comparing the two forms of $\left|\psi_{1}\right\rangle$, it suffices to show

$$
P_{4}\left(P_{3}^{(1)}-P_{3}^{(0)}\right)|\psi\rangle=Q_{4}\left(Q_{3}^{(0)}-Q_{3}^{(1)}\right)|\psi\rangle .
$$

This equation can be derived in the following way

$$
\begin{aligned}
& P_{4}\left(P_{3}^{(1)}-P_{3}^{(0)}\right)|\psi\rangle \\
= & P_{4}\left(P_{3}^{(1)}-P_{3}^{(0)}\right) P_{0}^{(0)}|\psi\rangle \\
= & \left(Q_{3}^{(1)}-Q_{3}^{(0)}\right) Q_{4} Q_{0}^{(0)}|\psi\rangle \\
= & Q_{4}\left(Q_{3}^{(0)}-Q_{3}^{(1)}\right) Q_{0}^{(0)}|\psi\rangle \\
= & Q_{4}\left(Q_{3}^{(0)}-Q_{3}^{(1)}\right)|\psi\rangle,
\end{aligned}
$$

where we use the fact that Equation (10) is satisfied in the inducing strategies $S$ and $S^{\prime}$. In the end, we apply Proposition 3.1 to $S^{\prime}$ to see that

$$
\begin{aligned}
& P_{1} P_{2}\left|\psi_{1}\right\rangle \\
= & \left(\mathbb{I}+i Q_{4}\right) P_{1} P_{2}\left(Q_{3}^{(0)}-i Q_{4} Q_{3}^{(1)}\right)|\psi\rangle \\
= & \omega_{p}^{-1}\left|\psi_{1}\right\rangle,
\end{aligned}
$$

which completes the proof.

\section{The self-testing correlation}

In this section, we introduce the correlation $P_{p, r}$ : $[n(r)+m(r)+3] \times[n(r)+m(r)+3] \times[8] \times[8] \rightarrow \mathbb{R}$, which can be thought of as the combination of $P_{\hat{A}}$ and $\hat{P}_{-\pi / p}$.

We define $P_{p, r}$ by giving the inducing strategy, which is based on $S_{\hat{A}}$ of $P_{\hat{A}}$ and $S_{-\pi / p}$ of $\hat{P}_{-\pi / p}$. Let

$$
\begin{aligned}
& \tilde{P}_{x}^{(a)}=\left\{\begin{array}{l}
\Pi_{x}^{(a)} \\
P_{x-n(r)}^{(a)} \\
\bar{P}_{0}^{(a)} \otimes \mathbb{I}_{\mathbb{C}^{2}}^{\otimes 2} \\
\bar{P}_{x+2-n(r)-m(r)}^{(a)} \otimes \mathbb{I}_{\mathbb{C}^{2}}^{\otimes 2} \\
0
\end{array}\right. \\
& \tilde{Q}_{y}^{(b)}=\left\{\begin{array}{l}
\Pi_{y}^{(b)} \\
P_{y}^{(b)} \\
\bar{Q}_{0}^{(b)} \otimes(r) \\
\bar{Q}_{y+2-n(r)-m(r)}^{(b)} \otimes \mathbb{I}_{\mathbb{C}^{2}}^{\otimes 2} \\
0
\end{array}\right.
\end{aligned}
$$

if $x \in[n(r)]$ and $a \in[2]$,

if $n(r) \leq x \leq n(r)+m(r)-1$,

if $x=n(r)+m(r)$ and $a \in[2]$,

if $x=n(r)+m(r)+1, n(r)+m(r)+2$ and $a \in[3]$,

otherwise;

if $y \in[n(r)]$ and $b \in[2]$,

if $n(r) \leq y \leq n(r)+m(r)-1$,

if $y=n(r)+m(r)$ and $b \in[2]$,

if $y=n(r)+m(r)+1, n(r)+m(r)+2$ and $b \in[3]$,

otherwise. 
The inducing strategy is

$$
\tilde{S}=\left(|\tilde{\psi}\rangle \otimes|E P R\rangle^{\otimes 2},\left\{\left\{\tilde{P}_{x}^{(a)} \mid a \in[8]\right\} \mid x \in[n(r)+m(r)+3]\right\},\left\{\left\{\tilde{Q}_{y}^{(b)} \mid b \in[8]\right\} \mid y \in[n(r)+m(r)+3]\right\}\right) .
$$

Note that in the definition of $\tilde{S}$, a bijection between $[n(r)]$ and $S_{\Gamma}$ is implicit.

Definition 4.1. The correlation $P_{p, r}:[n(r)+$ $m(r)+3] \times[n(r)+m(r)+3] \times[8] \times[8] \rightarrow \mathbb{R} i s$ induced by $\tilde{S}$ as

$$
\begin{aligned}
& P_{p, r}(a, b \mid x, y) \\
= & {\left[\left\langle\tilde { \psi } \left|\otimes\left\langle\left. E P R\right|^{\otimes 2}\right] \tilde{P}_{x}^{(a)} \tilde{Q}_{y}^{(b)}\left[|\tilde{\psi}\rangle \otimes|E P R\rangle^{\otimes 2}\right] .\right.\right.\right.}
\end{aligned}
$$

It is not hard to see that

- When $x \in[n(r)], n(r) \leq y \leq n(r)+m(r)-1$ and $a \in[2]$,

$$
P_{p, r}(a, b \mid x, y)=P_{\hat{A}}(b, a \mid y-n(r), x) .
$$

- When $n(r) \leq x \leq n(r)+m(r)-1, y \in[n(r)]$ and $b \in[2]$,

$$
P_{p, r}(a, b \mid x, y)=P_{\hat{A}}(a, b \mid x-n(r), y) .
$$

- When $x=y \in[n(r)]$,

$$
P_{p, r}(0,0 \mid x, x)+P_{p, r}(1,1 \mid x, x)=1 .
$$

- When $x, y \in\{0,1, n(r)+m(r), n(r)+m(r)+$ $1, n(r)+m(r)+2\}$, define $f:\{0,1, n(r)+$ $m(r), n(r)+m(r)+1, n(r)+m(r)+2\} \rightarrow[5]$ by

$$
f(x)=\left\{\begin{array}{l}
x+1 \text { if } x=0,1, \\
x-n(r)-m(r) \text { if } x=n(r)+m(r), \\
x+2-n(r)-m(r) \text { otherwise. }
\end{array}\right.
$$

Then, if $a, b \in[3]$

$$
P_{p, r}(a, b \mid x, y)=\hat{P}_{-\pi / p}(a, b \mid f(x), f(y)) .
$$

\section{Proposition 4.2. If a strategy}

$$
\begin{aligned}
S= & \left(|\psi\rangle \in \mathcal{H}_{A} \otimes \mathcal{H}_{B},\right. \\
& \left\{\left\{P_{x}^{(a)} \mid a \in[8]\right\} \mid x \in[n(r)+m(r)+3]\right\}, \\
& \left.\left\{\left\{Q_{y}^{(b)} \mid b \in[8]\right\} \mid y \in[n(r)+m(r)+3]\right\}\right)
\end{aligned}
$$

induces $P_{p, r}$, then there exist unitaries $U_{A} \in$ $\mathcal{U}\left(\mathcal{H}_{A}\right)$ and $U_{B} \in \mathcal{U}\left(\mathcal{H}_{B}\right)$ and a subnormalized state $\left|\psi_{1}\right\rangle \in \mathcal{H}_{A} \otimes \mathcal{H}_{B}$ such that

$$
\begin{aligned}
& \|\left|\psi_{1}\right\rangle \|^{2}=\frac{1}{p-1} \\
& |\psi\rangle=\sum_{j=1}^{(p-1)}\left(U_{A}^{\dagger} U_{B}^{\dagger}\right)^{\log _{r} j}\left|\psi_{1}\right\rangle
\end{aligned}
$$

where $\log _{r} j$ is the discrete log.

Before we prove the proposition, we fix some notations used in the proofs of this proposition and the next theorem. We relabel $P_{0}^{(a)}$ and $Q_{0}^{(b)}$ as $P_{o_{1}}^{(a)}$ and $Q_{o_{1}}^{(b)}$, and relabel $P_{1}^{(a)}$ and $Q_{1}^{(b)}$ as $P_{o_{2}}^{(a)}$ and $Q_{o_{2}}^{(b)}$. We can also identify variables of $\hat{A} x=0$ corresponding to the generators $u_{1}$ and $u_{2}$, so we label the corresponding Alice and Bob's projectors as $P_{u_{1}}^{(a)}, P_{u_{2}}^{(a)}$ and $Q_{u_{1}}^{(b)}, Q_{u_{2}}^{(b)}$. For $s \in\left\{u_{1}, u_{2}, o_{1}, o_{2}\right\}$, we further define

$$
P_{s}:=P_{s}^{(0)}-P_{s}^{(1)} \quad Q_{s}=Q_{s}^{(0)}-Q_{s}^{(1)},
$$

and

$$
\begin{array}{ll}
O_{A}:=P_{o_{1}} P_{o_{2}} & O_{B}:=Q_{o_{1}} Q_{o_{2}}, \\
U_{A}:=P_{u_{1}} P_{u_{2}} & U_{B}:=Q_{u_{1}} Q_{u_{2}} .
\end{array}
$$

Proof. Define $P_{n(r)+m(r)+1}:=P_{n(r)+m(r)+1}^{(0)}-$ $P_{n(r)+m(r)+1}^{(1)}, \quad P_{n(r)+m(r)+2}:=P_{n(r)+m(r)+2}^{(0)}-$ $P_{n(r)+m(r)+2}^{(1)}$, and

$$
\begin{aligned}
\left|\psi_{1}\right\rangle= & \frac{1}{2}\left(P_{n(r)+m(r)+1}^{(0)}+P_{n(r)+m(r)+1}^{(1)}\right. \\
& \left.-i P_{n(r)+m(r)+2} P_{n(r)+m(r)+1}\right)|\psi\rangle
\end{aligned}
$$

Since $S$ can induce $\hat{P}_{-\pi / p}$, by Proposition 3.3, we know $\|\left|\psi_{1}\right\rangle \|=1 / \sqrt{p-1}$, and

$$
O_{A}\left|\psi_{1}\right\rangle=\omega_{p}^{-1}\left|\psi_{1}\right\rangle, \quad O_{B}\left|\psi_{1}\right\rangle=\omega_{p}\left|\psi_{1}\right\rangle .
$$

Since $S$ can also induce $P_{\hat{A}}$, and $P_{\hat{A}}$ with Alice and Bob's roles flipped, by Proposition 2.4, we know

$$
\begin{aligned}
& U_{A} O_{A} U_{A}^{\dagger}|\psi\rangle=O_{A}^{r}|\psi\rangle, \\
& U_{B} O_{B} U_{B}^{\dagger}|\psi\rangle=O_{B}^{r}|\psi\rangle .
\end{aligned}
$$


By substitution and Lemma 2.5, we know

$$
\begin{aligned}
& O_{A}\left(U_{A}^{\dagger}\right)^{j}|\psi\rangle=\left(U_{A}^{\dagger}\right)^{j} O_{A}^{r^{j}}|\psi\rangle, \\
& O_{B}\left(U_{B}^{\dagger}\right)^{j}|\psi\rangle=\left(U_{B}^{\dagger}\right)^{j} O_{B}^{r j}|\psi\rangle,
\end{aligned}
$$

for $j \in[p-1]$. Then,

$$
\begin{aligned}
& O_{A}\left(U_{A}^{\dagger}\right)^{j}\left|\psi_{1}\right\rangle=\left(U_{A}^{\dagger}\right)^{j} O_{A}^{r^{j}}\left|\psi_{1}\right\rangle=\omega_{p}^{-r^{j}}\left(U_{A}^{\dagger}\right)^{j}\left|\psi_{1}\right\rangle, \\
& O_{B}\left(U_{B}^{\dagger}\right)^{j}\left|\psi_{1}\right\rangle=\left(U_{B}^{\dagger}\right)^{j} O_{B}^{r^{j}}\left|\psi_{1}\right\rangle=\omega_{p}^{r^{j}}\left(U_{B}^{\dagger}\right)^{j}\left|\psi_{1}\right\rangle,
\end{aligned}
$$

where we use the fact that $\left|\psi_{1}\right\rangle$ can be expressed using Alice's projectors and Bob's projectors as shown in Equations (23) and (27). Hence, $\left\{\left(U_{A}^{\dagger} U_{B}^{\dagger}\right)^{j}\left|\psi_{1}\right\rangle \mid j \in[p-1]\right\}$ is an orthogonal set and

$$
\| \sum_{j \in[p-1]}\left(U_{A}^{\dagger} U_{B}^{\dagger}\right)^{j}\left|\psi_{1}\right\rangle \|=1
$$

The last step is to check that $\left\langle\psi\left|\sum_{j \in[p-1]}\left(U_{A}^{\dagger} U_{B}^{\dagger}\right)^{j}\right| \psi_{1}\right\rangle=1$. Using the fact that $U_{A} U_{B}|\psi\rangle=P_{u_{1}} Q_{u_{1}} P_{u_{2}} Q_{u_{2}}|\psi\rangle=|\psi\rangle$, we can see that

$$
\left\langle\psi\left|\sum_{j \in[p-1]}\left(U_{A}^{\dagger} U_{B}^{\dagger}\right)^{j}\right| \psi_{1}\right\rangle=(p-1)\left\langle\psi \mid \psi_{1}\right\rangle .
$$

Hence, the problem is reduced to calculate $\left\langle\psi \mid \psi_{1}\right\rangle$, which is

$$
\begin{aligned}
& \frac{1}{2}\langle\psi|\left(P_{n(r)+m(r)+1}^{(0)}+P_{n(r)+m(r)+1}^{(1)}\right. \\
& \left.-i P_{n(r)+m(r)+2} P_{n(r)+m(r)+1}\right)|\psi\rangle \\
= & \frac{1}{p-1}-\frac{i}{2}\left\langle\psi\left|Q_{n(r)+m(r)+2} P_{n(r)+m(r)+1}\right| \psi\right\rangle \\
= & \frac{1}{p-1},
\end{aligned}
$$

where $\left\langle\psi\left|Q_{n(r)+m(r)+2} P_{n(r)+m(r)+1}\right| \psi\right\rangle=0$ comes from the correlation. Then the proposition follows.

Theorem 4.3. Let $S$ be an inducing strategy of $P_{p, r}$ with a shared state $|\psi\rangle$. Then there exist an isometry $\Phi_{A} \otimes \Phi_{B}$ and a state $|j u n k\rangle$ such that

$$
\begin{aligned}
& \left.\Phi_{A} \otimes \Phi_{B}(|\psi\rangle)=\mid j \text { unk }\right\rangle \otimes|\tilde{\psi}\rangle \\
& \Phi_{A} \otimes \Phi_{B}\left(O_{A}|\psi\rangle\right)=|j u n k\rangle \otimes\left[\left(O_{1} O_{2}\right) \otimes \mathbb{I}\right]|\tilde{\psi}\rangle \\
& \Phi_{A} \otimes \Phi_{B}\left(O_{B}|\psi\rangle\right)=|j u n k\rangle \otimes\left[\mathbb{I} \otimes\left(O_{1} O_{2}\right)\right]|\tilde{\psi}\rangle \\
& \Phi_{A} \otimes \Phi_{B}\left(U_{A}|\psi\rangle\right)=|j u n k\rangle \otimes\left[\left(U_{1} U_{2}\right) \otimes \mathbb{I}\right]|\tilde{\psi}\rangle \\
& \Phi_{A} \otimes \Phi_{B}\left(U_{B}|\psi\rangle\right)=|j u n k\rangle \otimes\left[\mathbb{I} \otimes\left(U_{1} U_{2}\right)\right]|\tilde{\psi}\rangle,
\end{aligned}
$$

where $|\tilde{\psi}\rangle$ is defined in Equation (20) and $\mathrm{O}_{1}, \mathrm{O}_{2}, U_{1}, U_{2}$ are defined in Equations (16) to (19) respectively.
The isometry $\Phi_{A} \otimes \Phi_{B}$ is given in the figure below. It is designed based on the swap isometry proposed in [30].

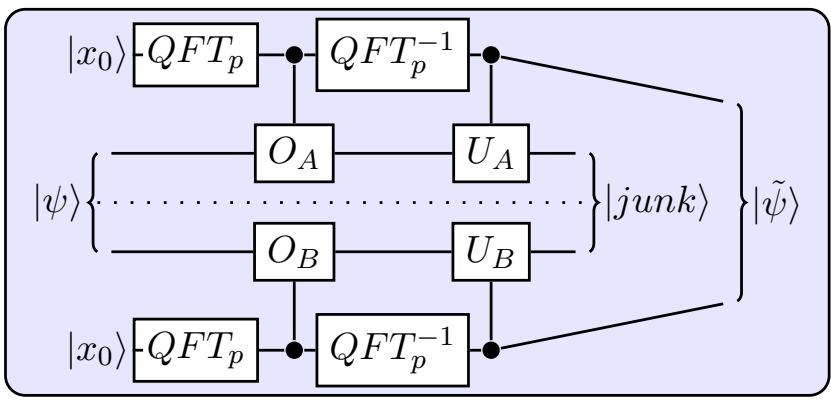

Figure 1: The isometries $\Phi_{A} \otimes \Phi_{B}$.

The isometry $\Phi_{A} \otimes \Phi_{B}$ has the following steps:

1. Append control register $\left|x_{0}\right\rangle_{A^{\prime}}$ on Alice's side and $\left|x_{0}\right\rangle_{B^{\prime}}$ on Bob's side, where $\left|x_{0}\right\rangle$ is orthogonal to $\mathbb{C}^{p-1}$;

2. Apply Quantum Fourier Transform $\left(Q F T_{p}\right)$ to Alice and Bob's control registers;

3. Apply Controlled- $O_{A / B}$ operations (i.e. if the control register is in state $\left|x_{k}\right\rangle_{A^{\prime} / B^{\prime}}$, apply $O_{A / B}^{k}$.);

4. Apply inverse Quantum Fourier Transform $\left(Q F T_{p}^{-1}\right)$ to the control registers;

5. Apply Controlled- $U_{A / B}$ operations (i.e. If Alice's control register is in state $\left|x_{j}\right\rangle$, she applies $U_{A}^{\log _{r}(p-j)}$. If Bob's control register is in state $\left|x_{j}\right\rangle$, he applies $\left.\left(U_{B}\right)^{\log _{r} j}\right)$.

Proof. Proposition 4.2 implies that

$$
\Phi_{A} \otimes \Phi_{B}(|\psi\rangle)=\Phi_{A} \otimes \Phi_{B}\left(\sum_{j=1}^{(p-1)}\left(U_{A}^{\dagger} U_{B}^{\dagger}\right)^{\log _{r} j}\left|\psi_{1}\right\rangle\right)
$$

Then the state $|\psi\rangle$ is evolved by the isometry as 
follows

$$
\begin{aligned}
& \sum_{j=1}^{p-1}\left(U_{A}^{\dagger} U_{B}^{\dagger}\right)^{\log _{r} j}\left|\psi_{1}\right\rangle\left|x_{0}\right\rangle_{A^{\prime}}\left|x_{0}\right\rangle_{B^{\prime}} \\
& \stackrel{Q F T_{p}}{\longrightarrow} \frac{1}{p} \sum_{k_{1}, k_{2} \in[p]} \sum_{j=1}^{p-1}\left(U_{A}^{\dagger} U_{B}^{\dagger}\right)^{\log _{r} j} \\
& \times\left|\psi_{1}\right\rangle\left|x_{k_{1}}\right\rangle_{A^{\prime}}\left|x_{k_{2}}\right\rangle_{B^{\prime}} \\
& \stackrel{\text { Controlled- } O_{A / B}}{\longrightarrow} \frac{1}{p} \sum_{k_{1}, k_{2} \in[p]} \sum_{j=1}^{p-1} O_{A}^{k_{1}}\left(U_{A}^{\dagger}\right)^{\log _{r} j} \\
& \times O_{B}^{k_{2}}\left(U_{B}^{\dagger}\right)^{\log _{r} j}\left|\psi_{1}\right\rangle\left|x_{k_{1}}\right\rangle_{A^{\prime}}\left|x_{k_{2}}\right\rangle_{B^{\prime}} \\
& =\frac{1}{p} \sum_{k_{1}, k_{2} \in[p]} \sum_{j=1}^{p-1}\left(U_{A}^{\dagger} U_{B}^{\dagger}\right)^{\log _{r} j} \\
& \times \omega_{p}^{\left(k_{2}-k_{1}\right) j}\left|\psi_{1}\right\rangle\left|x_{k_{1}}\right\rangle_{A^{\prime}}\left|x_{k_{2}}\right\rangle_{B^{\prime}} \\
& \stackrel{Q F T_{p}^{-1}}{\longrightarrow} \frac{1}{p^{2}} \sum_{j=1}^{p-1} \sum_{l_{1}, l_{2} \in[p]}\left(U_{A}^{\dagger} U_{B}^{\dagger}\right)^{\log _{r} j} \\
& \times\left(\sum_{k_{1} \in[p]} \omega_{p}^{k_{1}\left(p-j-l_{1}\right)}\right) \\
& \times\left(\sum_{k_{2} \in[p]} \omega_{p}^{k_{2}\left(j-l_{2}\right)}\right)\left|\psi_{1}\right\rangle\left|x_{l_{1}}\right\rangle_{A^{\prime}}\left|x_{l_{2}}\right\rangle_{B^{\prime}} \\
& =\sum_{j=1}^{p-1}\left(U_{A}^{\dagger} U_{B}^{\dagger}\right)^{\log _{r} j}\left|\psi_{1}\right\rangle\left|x_{p-j}\right\rangle_{A^{\prime}}\left|x_{j}\right\rangle_{B^{\prime}} \\
& \stackrel{\text { Controlled- } U_{A / B}}{\longrightarrow} \sum_{j=1}^{p-1} U_{A}^{\log _{r} j}\left(U_{A}^{\dagger}\right)^{\log _{r} j} U_{B}^{\log _{r} j}\left(U_{B}^{\dagger}\right)^{\log _{r} j} \\
& \times\left|\psi_{1}\right\rangle\left|x_{p-j}\right\rangle_{A^{\prime}}\left|x_{j}\right\rangle_{B^{\prime}} \\
& =\left|\psi_{1}\right\rangle \otimes \sum_{j=1}^{p-1}\left|x_{p-j}\right\rangle_{A^{\prime}}\left|x_{j}\right\rangle_{B^{\prime}}
\end{aligned}
$$

where we use the fact that for $l \in[p]$,

$$
\sum_{k \in[p]} \omega_{p}^{k(j-l)}= \begin{cases}p & \text { if } l=j \\ 0 & \text { otherwise. }\end{cases}
$$

The derivations of the other four equations follow very similar argument, so instead of giving the full proof, we give the key steps for them. For $O_{A}$ and $O_{B}$, we use the fact that

$$
\begin{aligned}
O_{A} \sum_{j=1}^{p-1}\left(U_{A}^{\dagger} U_{B}^{\dagger}\right)^{\log _{r} j}\left|\psi_{1}\right\rangle & =\sum_{j=1}^{p-1} \omega_{p}^{-j}\left(U_{A}^{\dagger} U_{B}^{\dagger}\right)^{\log _{r} j}\left|\psi_{1}\right\rangle, \\
O_{B} \sum_{j=1}^{p-1}\left(U_{A}^{\dagger} U_{B}^{\dagger}\right)^{\log _{r} j}\left|\psi_{1}\right\rangle & =\sum_{j=1}^{p-1} \omega_{p}^{j}\left(U_{A}^{\dagger} U_{B}^{\dagger}\right)^{\log _{r} j}\left|\psi_{1}\right\rangle .
\end{aligned}
$$

For $U_{A}$ and $U_{B}$, we use the fact that

$$
\begin{aligned}
& O_{A}\left(U_{A}^{\dagger}\right)^{\log _{r} j-1}\left|\psi_{1}\right\rangle=\omega_{p}^{-j r^{-1}}\left(U_{A}^{\dagger}\right)^{\log _{r} j-1}\left|\psi_{1}\right\rangle, \\
& O_{B}\left(U_{B}^{\dagger}\right)^{\log _{r} j-1}\left|\psi_{1}\right\rangle=\omega_{p}^{j r^{-1}}\left(U_{B}^{\dagger}\right)^{\log _{r} j-1}\left|\psi_{1}\right\rangle,
\end{aligned}
$$

for all $1 \leq j \leq p-1$.

Theorem 4.3 implies that, for any odd prime number $p$ whose primitive root is $r$, there exists a correlation of size $\Theta\left(r^{2}\right)$ that can self-test a maximally entangled state of local dimension $p-1$. Since there exists $r \in\{2,3,5\}$ such that there are infinitely many prime numbers whose smallest primitive root is $r$ [21], we can apply Theorem 4.3 to the set $D$ of all such odd prime numbers and obtain Theorem 1.

\section{Conclusion and discussions}

We have shown that there exists a family of constant-sized correlations such that each correlation of this family can self-test a maximally entangled state of a different dimension.

Curious readers may wonder if we can self-test $|\tilde{\psi}\rangle \otimes|E P R\rangle^{\otimes 2}$ since the inducing strategy uses it. Indeed, we can self-test it, but the proof of the self-test requires more details of the representation of $\Gamma(\hat{A})$ and modifying $\hat{A} x=0$ to introduce equations of the Magic square game. Since the techniques to self-test $|E P R\rangle^{\otimes 2}$ is standard in the literature, we refer to [11, Section 6], which is the previous arXiv version of this paper, for details of self-testing $|\tilde{\psi}\rangle \otimes|E P R\rangle^{\otimes 2}$.

The other question to ask is if we can self-test $O_{1}$ and $O_{2}$ individually. We don't have an answer for this question. The progress of answering this question is summarized here. Following the calculation in the proof of Proposition 3.3, we can see that

$$
\begin{aligned}
& \left(P_{1} P_{2}\right) P_{2}\left|\psi_{1}\right\rangle=\omega_{p} P_{2}\left|\psi_{1}\right\rangle, \\
& \left(Q_{1} Q_{2}\right) Q_{2}\left|\psi_{1}\right\rangle=\omega_{p}^{-1} Q_{2}\left|\psi_{1}\right\rangle .
\end{aligned}
$$

Then, to self-test $O_{1}$ and $O_{2}$ on Alice's side, we need to modify the controlled- $U_{A / B}$ step so that when the control-register is in the state $\left|x_{j}\right\rangle$, both Alice and Bob apply $U_{A / B}^{\log _{r} j}$. If we denote the modified isometry by $\Phi_{A}^{\prime} \otimes \Phi_{B}^{\prime}$,

$$
\Phi_{A}^{\prime} \otimes \Phi_{B}^{\prime}\left(P_{o_{x}}|\psi\rangle\right)=|j u n k\rangle \otimes\left(O_{x} \otimes \mathbb{I}\right)|\tilde{\psi}\rangle,
$$

with $x=1,2$. What is interesting is that if we want to self-test $O_{1}$ and $O_{2}$ on Bob's side, we need 
to modify the controlled- $U_{A / B}$ step again, so that when the control-register is in the state $\left|x_{j}\right\rangle$, both Alice and Bob apply $U_{A / B}^{\log _{r}(p-j)}$. If we denote the modified isometry by $\Phi_{A}^{\prime \prime} \otimes \Phi_{B}^{\prime \prime}$,

$$
\Phi_{A}^{\prime \prime} \otimes \Phi_{B}^{\prime \prime}\left(Q_{o_{y}}|\psi\rangle\right)=|j u n k\rangle \otimes\left(\mathbb{I} \otimes O_{y}\right)|\tilde{\psi}\rangle,
$$

with $y=1,2$. Since $\Phi_{A}^{\prime} \otimes \Phi_{B}^{\prime}$ and $\Phi_{A}^{\prime \prime} \otimes \Phi_{B}^{\prime \prime}$ are different from $\Phi_{A} \otimes \Phi_{B}$, we cannot conclude that $P_{p, r}$ is a self-test of $|\tilde{\psi}\rangle$ and the binary observables $O_{1}$ and $O_{2}$. On the other hand, it has been shown projectors of arbitrary rank can be self-tested [17]. If we can self-test $O_{1}, O_{2}$ and even $U_{1}$ and $U_{2}$, we can get a second self-test of projectors of arbitrary rank.

The last question about $P_{p, r}$ is about its robustness. In the arXiv version [11], we have shown that if a quantum strategy $S$ can induce a correlation $P^{\prime}$ such that

$$
\max _{x, y, a, b}\left|P^{\prime}(a, b \mid x, y)-P_{p, r}(a, b \mid x, y)\right| \leq \epsilon,
$$

then,

$$
\| \Phi_{A} \otimes \Phi_{B}(|\psi\rangle)-|j u n k\rangle \otimes|\tilde{\psi}\rangle \|=O\left(r^{p} \epsilon^{1 / 8}\right) .
$$

The derivation of the robustness is very long and technical, so we choose the journal version to focus on illustrating the basic ideas. Improving the robustness bound is another open problem about $P_{p, r}$.

\section{Acknowledgment}

The author is deeply grateful to his $\mathrm{PhD}$ advisor, Carl Miller, for the enlightening discussions with him. Carl's original ideas about combining a linear system game with a Bell inequality and about characterizing prime numbers by their primitive roots are invaluable to this work. Carl also helped the author with the design of $\hat{P}_{-\pi / p}$.

\section{References}

[1] Antonio Acín, Serge Massar, and Stefano Pironio. Randomness versus nonlocality and entanglement. Physical review letters, 108(10):100402, 2012. DOI: https://doi.org/10.1103/PhysRevLett.108.100402.

[2] Padmanabhan K. Aravind. Quantum mysteries revisited again. American Journal of Physics, 72(10):1303-1307, 2004. DOI: https://doi.org/10.1119/1.1773173.
[3] Cédric Bamps and Stefano Pironio. Sumof-squares decompositions for a family of Clauser-Horne-Shimony-Holt-like inequalities and their application to self-testing. Physical Review A, 91(5):052111, 2015. DOI: https://doi.org/10.1103/PhysRevA.91.052111.

[4] Spencer Breiner, Amir Kalev, and Carl Miller. Parallel self-testing of the GHZ state with a proof by diagrams. In 15th International Conference on Quantum Physics and Logic; June 3-7, 2018; Halifax, Canada, volume 287, pages 43-66, 2018. DOI: https://doi.org/10.4204/EPTCS.287.3.

[5] John F Clauser, Michael A Horne, Abner Shimony, and Richard A Holt. Proposed experiment to test local hidden-variable theories. Physical review letters, 23(15):880, $1969 . \quad$ DOI: https://doi.org/10.1103/PhysRevLett.23.880.

[6] Richard Cleve, Li Liu, and William Slofstra. Perfect commuting-operator strategies for linear system games. Journal of Mathematical Physics, 58(1):012202, 2017. DOI: https://doi.org/10.1063/1.4973422.

[7] Andrea Coladangelo and Jalex Stark. Robust self-testing for linear constraint system games. arXiv preprint arXiv:1709.09267, 2017.

[8] Andrea Coladangelo, Koon Tong Goh, and Valerio Scarani. All pure bipartite entangled states can be self-tested. $\mathrm{Na}$ ture communications, 8:15485, 2017. DOI: https://doi.org/10.1038/ncomms15485.

[9] Andrea Coladangelo, Alex B Grilo, Stacey Jeffery, and Thomas Vidick. Verifier-on-aleash: new schemes for verifiable delegated quantum computation, with quasilinear resources. In Annual International Conference on the Theory and Applications of Cryptographic Techniques, pages 247-277. Springer, 2019. DOI: https://doi.org/10.1007/978-3030-17659-4_9.

[10] Joseph Fitzsimons, Zhengfeng Ji, Thomas Vidick, and Henry Yuen. Quantum proof systems for iterated exponential time, and beyond. In Proceedings of the 51st Annual ACM SIGACT Symposium on Theory of Computing, pages 473-480. ACM, 2019. DOI: https://doi.org/10.1145/3313276.3316343.

[11] Honghao Fu. Constant-sized correlations are sufficient to robustly self-test maximally en- 
tangled states with unbounded dimension. arXiv preprint arXiv:1911.01494, 2019.

[12] Honghao $\mathrm{Fu}$ and Carl A Miller. Local randomness: Examples and application. Physical Review A, 97(3):032324, 2018. DOI: https://doi.org/10.1103/PhysRevA.97.032324.

[13] D. R. Heath-Brown. Artin's conjecture for primitive roots. The Quarterly Journal of Mathematics, 37(1): 27-38, 03 1986. ISSN 0033-5606. DOI: https://doi.org/10.1093/qmath/37.1.27.

URL https://doi.org/10.1093/qmath/ 37.1.27.

[14] Zhengfeng Ji, Anand Natarajan, Thomas Vidick, John Wright, and Henry Yuen. $M I P^{*}=R E$. arXiv preprint arXiv:2001.04383, 2020.

[15] Jȩdrzej Kaniewski. Self-testing of binary observables based on commutation. Physical Review A, 95(6):062323, 2017. DOI: https://doi.org/10.1103/PhysRevA.95.062323. URL https://link.aps.org/doi/10. 1103/PhysRevA.95.062323.

[16] Thomas Lawson, Noah Linden, and Sandu Popescu. Biased nonlocal quantum games. arXiv preprint arXiv:1011.6245, 2010.

[17] Laura Mančinska, Jitendra Prakash, and Christopher Schafhauser. Constant-sized robust self-tests for states and measurements of unbounded dimension. arXiv preprint arXiv:2103.01729, 2021.

[18] Dominic Mayers and Andrew Yao. Quantum cryptography with imperfect apparatus. In Proceedings 39th Annual Symposium on Foundations of Computer Science (Cat. No. 98CB36280), pages 503-509. IEEE, 1998. DOI: https://doi.org/10.1109/SFCS.1998.743501.

[19] Matthew McKague, Tzyh Haur Yang, and Valerio Scarani. Robust self-testing of the singlet. Journal of Physics A: Mathematical and Theoretical, 45(45):455304, 2012. DOI: https://doi.org/10.1088/1751$8113 / 45 / 45 / 455304$.

[20] N. David Mermin. Simple unified form for the major no-hiddenvariables theorems. Phys. Rev. Lett., 65:3373-3376, Dec 1990. DOI: https://doi.org/10.1103/PhysRevLett.65.3373. URL https://link.aps.org/doi/10. 1103/PhysRevLett.65.3373.
[21] Maruti Ram Murty. Artin's conjecture for primitive roots. The Mathematical Intelligencer, 10(4):59-67, $1988 . \quad$ DOI: https://doi.org/10.1007/BF03023749.

[22] Anand Natarajan and Thomas Vidick. A quantum linearity test for robustly verifying entanglement. In Proceedings of the 49th Annual ACM SIGACT Symposium on Theory of Computing, pages 1003-1015. ACM, 2017. DOI: https://doi.org/10.1145/3055399.3055468.

[23] Anand Natarajan and Thomas Vidick. Lowdegree testing for quantum states, and a quantum entangled games $P C P$ for QMA. In 2018 IEEE 59th Annual Symposium on Foundations of Computer Science (FOCS), pages 731-742. IEEE, 2018. DOI: https://doi.org/10.1109/FOCS.2018.00075.

[24] Anand Natarajan and John Wright. $N E E X P$ is Contained in $M I P^{*}$. In 2019 IEEE 60th Annual Symposium on Foundations of Computer Science (FOCS), pages 510-518. IEEE, 2019. DOI: https://doi.org/10.1109/FOCS.2019.00039.

[25] Ben W Reichardt, Falk Unger, and Umesh Vazirani. Classical command of quantum systems. Nature, 496(7446):456, 2013. DOI: https://doi.org/10.1038/nature12035.

[26] Joseph J Rotman. An introduction to the theory of groups, volume 148. Springer Science \& Business Media, 2012. DOI: https://doi.org/10.1007/978-1-4612-4176-8.

[27] Shubhayan Sarkar, Debashis Saha, Jędrzej Kaniewski, and Remigiusz Augusiak. Selftesting quantum systems of arbitrary local dimension with minimal number of measurements. npj Quantum Information, 7(1):151, 2021. DOI: https://doi.org/10.1038/s41534021-00490-3. URL https://doi.org/10. 1038/s41534-021-00490-3.

[28] William Slofstra. The set of quantum correlations is not closed. In $F_{O}$ rum of Mathematics, Pi, volume 7. Cambridge University Press, 2019. DOI: https://doi.org/10.1017/fmp.2018.3.

[29] Xingyao Wu, Jean-Daniel Bancal, Matthew McKague, and Valerio Scarani. Device-independent parallel self-testing of two singlets. Physical Review A, 93(6):062121, 2016. DOI: https://doi.org/10.1103/PhysRevA.93.062121. 
[30] Tzyh Haur Yang and Miguel Navascués. Robust self-testing of unknown quantum systems into any entangled two-qubit states. Physical Review A, 87(5):050102, 2013. DOI: https://doi.org/10.1103/PhysRevA.87.050102. 